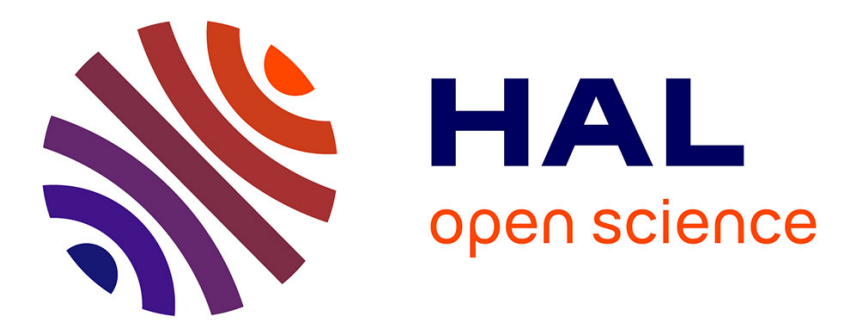

\title{
Caractérisation physico-chimique et électrique de structures fluorure - semi-conducteur III-V (passivation de GaAs et InP)
}

A.S. Barrière, A. Chaouki, G. Couturier, T. Seguelong, C. Sribi, P. Alnot

\section{To cite this version:}

A.S. Barrière, A. Chaouki, G. Couturier, T. Seguelong, C. Sribi, et al.. Caractérisation physicochimique et électrique de structures fluorure - semi-conducteur III-V (passivation de GaAs et InP). Revue de Physique Appliquée, 1988, 23 (1), pp.63-70. 10.1051/rphysap:0198800230106300 . jpa00245748

\section{HAL Id: jpa-00245748 https://hal.science/jpa-00245748}

Submitted on 1 Jan 1988

HAL is a multi-disciplinary open access archive for the deposit and dissemination of scientific research documents, whether they are published or not. The documents may come from teaching and research institutions in France or abroad, or from public or private research centers.
L'archive ouverte pluridisciplinaire HAL, est destinée au dépôt et à la diffusion de documents scientifiques de niveau recherche, publiés ou non, émanant des établissements d'enseignement et de recherche français ou étrangers, des laboratoires publics ou privés. 


\title{
Caractérisation physico-chimique et électrique de structures fluorure - semi-conducteur III-V (passivation de GaAs et InP) ( $\left.{ }^{1}\right)$
}

\author{
A. S. Barrière $\left({ }^{*}\right)$, A. Chaouki $(*)$, G. Couturier $(*)$, T. Seguelong $\left({ }^{*}\right)$, C. Sribi $\left({ }^{*}\right)$ et P. Alnot $\left({ }^{* *}\right)$ \\ (*) LEMME, 351, Cours de la Libération, 33405 Talence Cedex, France \\ (**) LCR THOMSON CSF, Domaine de Corbeville, 91401 Orsay, France
}

(Reçu le 16 janvier 1987, révisé le 15 septembre 1987, accepté le 17 septembre 1987)

\begin{abstract}
Résumé. - Les travaux présentés se rapportent à la caractérisation physico-chimique et électrique de couches minces de $\mathrm{SrF}_{2}$ déposées sous vide sur InP et de films d'InF$F_{3}$ et de $\mathrm{GaF}_{3}$ résultant d'une fluoruration partielle d'InP et de GaAs. Quel que soit le mode de préparation, à la précision de la R.B.S. près, le volume des couches de fluorure présente la stœchiométrie attendue et une étude X.P.S. révèle qu'aux interfaces couchessubstrats le phosphore et l'arsenic sont liés au fluor. Par ailleurs, dans tous les cas, les caractéristiques $C-V$ des structures M.I.S. obtenues montrent une importante modulation du potentiel de surface des semi-conducteurs. Dans le cas de $\mathrm{SrF}_{2}$-InP la densité d'états d'interface est très faible $\left(\simeq 10^{11} \mathrm{eV}^{-1} \mathrm{~cm}^{-2}\right)$ pour InP et GaAs fluorés elle est voisine de $10^{12}$ et $5 \times 10^{11} \mathrm{eV}^{-1} \mathrm{~cm}^{-2}$ respectivement.
\end{abstract}

\begin{abstract}
The presented results deal with the physico-chemical and electrical characterization of $\mathrm{SrF}_{2}$ thin films deposited under vacuum on $\mathrm{InP}$ and of $\mathrm{InF}_{3}$ and $\mathrm{GaF}_{3}$ layers resulting from a partial fluorination of InP and GaAs. Whatever the preparation mode may be, at the precision of the R.B.S., the bulk of the fluoride layers presents the expected stoichiometry and a X.P.S. study reveals that phosphorus and arsenic are bounded with fluorine at the film-substrate interfaces. In other respect, in all cases, the $C$ - $V$ characteristics of the obtained M.I.S. structures show an important modulation of the surface potential of the semiconductor. For $\mathrm{SrF}_{2}-\mathrm{InP}$ the interface state density is very low $\left(\simeq 10^{11} \mathrm{eV}^{-1} \mathrm{~cm}^{-2}\right)$; for the fluorinated InP and $\mathrm{GaAs}$ samples it is about $10^{12}$ and $5 \times 10^{11} \mathrm{eV}^{-1} \mathrm{~cm}^{-2}$ respectively.
\end{abstract}

\section{Introduction.}

Ces dernières années, de nombreux travaux ont été consacrés à la passivation des composés III-V. Si les premières publications mettaient en évidence une importante densité $\left(N_{\mathrm{SS}}\right)$ d'états localisés, situés dans la bande interdite des semi-conducteurs, près de l'interface [1-3], récemment, grâce en particulier à des traitements de surface, à des techniques douces de déposition ou d'« oxydation », de bien meilleurs résultats ont été obtenus, principalement avec InP et des isolants « classiques »: oxydes, nitrures, sulfures, ... Dans ce cas $N_{\mathrm{Ss}}$ est communément de l'ordre de $10^{12} \mathrm{eV}^{-1} \mathrm{~cm}^{-2}$. Une intéressante synthèse de ces travaux a fait l'objet des «Journées Epitaxie et Passivation des Composés III-V » [4].

(1) Cet article avait été proposé pour le numéro spécial Semi-conducteurs III-V. Sa parution a été retardée pour des raisons matérielles indépendantes de l'éditeur.
Par ailleurs, pour minimiser l'influence de la rupture de l'ordre atomique à l'interface semiconducteur-isolant, des solutions solides de fluorures d'éléments du groupe II-A (structure fluorine) ont pu être épitaxiées sur GaAs $[5,6]$ et InP $[7,8]$ (structure zinc-blende) permettant notamment d'envisager la réalisation de composants tridimensionnels.

Pour notre part, dans un premier temps, nous avons étudié les couples $\mathrm{Ba}_{0,175} \mathrm{Sr}_{0,825} \mathrm{~F}_{2} / \mathrm{InP}(100)$ et $\mathrm{Sr}_{0,565} \mathrm{Ca}_{0,435} \mathrm{~F}_{2} / \mathrm{GaAs}$ (100) obtenus par sublimation thermique sous vide secondaire des fluorures [9]. Dans le premier cas, nous avons montré que la sublimation de l'alliage n'étant pas congruente, conduit à une composition évolutive de l'isolant dans l'épaisseur des couches. Ce défaut favorise un transport ionique qui semble être responsable de la forte hystérésis observée sur les caractéristiques $C-V$ des structures MIS obtenues avec ces matériaux. Par contre dans le cas des structures $(\mathrm{SrCa}) \mathrm{F}_{2} / \mathrm{GaAs}$, malgré la qualité du diélectrique (quasi stœchiomé- 
trique, présentant une épitaxie partielle avec le semi-conducteur et de très faibles pertes), nous n'avons jamais observé de déblocage du niveau de Fermi ; ce défaut a été attribué à la difficulté de nettoyage et de conservation de la surface propre de GaAs, soit donc à la persistance de traces d'oxydes natifs à l'interface couche-substrat.

Pour tenter de corriger ces imperfections, dans le cas d'InP, tout en conservant la même technique de passivation, nous avons choisi de déposer un composé de formule chimique plus simple, $\mathrm{SrF}_{2}$, que nous pouvions espérer obtenir stœechiométrique ; dans l'autre cas nous avons préféré, par contre, réaliser les structures par fluoruration de GaAs. Cette opération, se traduisant par un déplacement de l'interface couche-substrat dans le volume de ce dernier, peut réduire la présence d'oxydes natifs à la surface du semi-conducteur dont les effets néfastes ne sont plus à démontrer. Nous remarquerons que cette technique peut également s'appliquer à la passivation d'InP.

Dans le présent article sont reportés les premiers résultats obtenus avec ces nouveaux matériaux.

\section{Etude des structures $\mathrm{SrF}_{2} / \mathrm{InP}(100)$.}

2.1 PréPARATION, CARACTÉRISATIONS PHYSICOCHIMIQUES. - Les poudres initiales de $\mathrm{SrF}_{2}$ (produits MERCK 99,9999) ont été refluorées sous fluor juste avant leur utilisation pour éliminer d'éventuelles traces d'oxygène ou de vapeur d'eau. La nature des substrats varie en fonction de l'étude envisagée. Dans le cas d'InP (100), après avoir subi un nettoyage standard (trichloroéthylène bouillant, $\mathrm{HF}(49 \%)-\mathrm{CH}_{3} \mathrm{OH}(1: 5)$ à $300 \mathrm{~K}$, méthanol chaud), les plaquettes sont maintenues sous atmosphère inerte $\left(\mathrm{Ar}, \mathrm{N}_{2}\right)$ jusqu'à leur mise sous vide. Les films sont déposés sous une pression résiduelle de $10^{-7} \mathrm{~T}$ à partir de creusets de platine chauffés par effet Joule. La température des substrats, $T_{\mathrm{S}}$, pendant la croissance des couches et leur vitesse de condensation, $V_{\mathrm{C}}$, sont maintenues constantes $\left(T_{\mathrm{S}}=573 \mathrm{~K}, V_{\mathrm{C}}=1 \AA \mathrm{s}^{-1}\right)$. L'épaisseur des films, évaluée lors de la croissance et contrôlée a posteriori, peut varier de 40 à $5000 \AA$.

Pour la caractérisation électrique des films dé $\mathrm{SrF}_{2}$, des structures sandwichs M.I.M. (Au$\mathrm{SrF}_{2}-\mathrm{Au}$ ) ont été réalisées sur substrats de verre sans passage à l'air grâce à un jeu de caches. Dans le cas des structures MIS un contact ohmique or-germanium est réalisé sur la face arrière du dispositif, le contact supérieur est assuré par une goutte de mercure.

Dans ces conditions une étude de diffraction $\mathrm{X}$ [10] montre que les films obtenus sont polycristallins et que la majorité des grains qui les constituent sont épitaxés sur InP. Ce résultat est la conséquence du faible écart relatif de maille entre le fluorure (struc- ture fluorine) et le semi-conducteur (structure zincblende) qui n'est que de $1,2 \%$.

La composition des couches minces de $\mathrm{SrF}_{2}$, déposées sur des substrats de carbone, a été principalement déduite d'études de diffusion Rutherford (R.B.S.) de particules $\alpha$ de $2 \mathrm{MeV}(*)$. A la précision des mesures près, en plus des éléments de base (strontium et fluor), seul de l'oxygène a été détecté. A partir de la section efficace de diffusion Rutherford des différents constituants et du pouvoir d'arrêt du matériau, nous avons pu établir les profils de concentration en fluor et en oxygène rapportés à un atome de strontium dans l'épaisseur des couches. Les résultats obtenus pour un film de $1200 \AA$ d'épaisseur sont reportés figure 1 , spectres $a$ et $b$ respectivement. On remarquera que dans le volume, et jusqu'à l'interface couche-substrat, le produit obtenu semble bien présenter la stœchiométrie attendue. La présence d'oxygène, uniquement détecté à la surface externe des échantillons, dont la teneur peut être considérablement réduite par un revêtement d'or, résulte vraisemblablement d'une adsorption de vapeur d'eau lors du transfert des cibles du bâti de préparation dans la chambre d'analyse, suivie d'une réaction chimique avec le fluorure conduisant à la formation de l'oxyfluorure $\mathrm{SrF}_{2-x} \mathrm{O}_{x / 2}$. Il est à noter que ce dernier doit luimême protéger le volume des couches d'une diffusion de vapeur d'eau puisque les profils obtenus restent constants dans le temps.

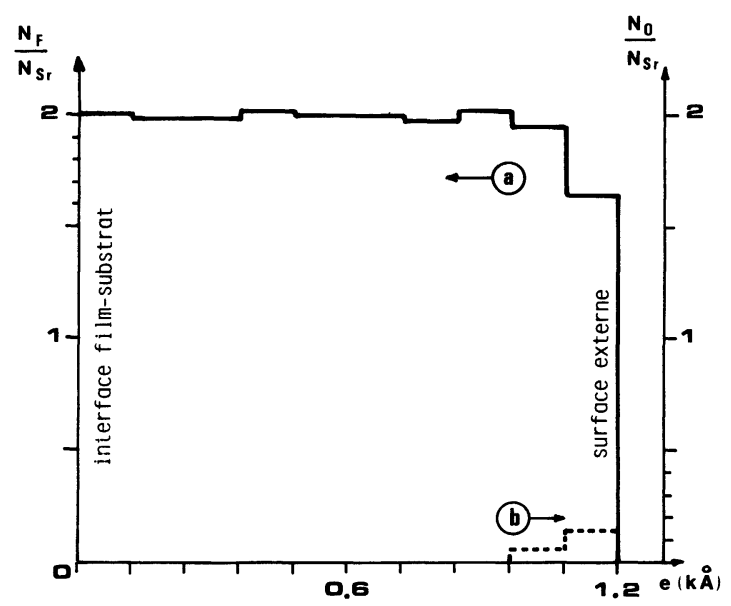

Fig. 1. - Profil de concentration en fluor (spectre a) et en oxygène (spectre b) d'un film de $\mathrm{SrF}_{2}$ de $1200 \AA$ d'épaisseur déposé à $573 \mathrm{~K}$ sur un substrat de carbone. Ces résultats sont déduits d'études R.B.S.

[R.B.S. fluorine and oxygen concentration profiles along the depth of a $1200 \AA$ thick $\mathrm{SrF}_{2}$ layer deposited on carbon substrate at $573 \mathrm{~K}$.]

(*) Cette étude est réalisée sur une ligne de l'accélérateur VDG $4 \mathrm{MeV}$ du Centre d'Etudes Nucléaires de Bordeaux-Gradignan, que nous avons équipée à des fins d'analyses. 
La caractérisation de l'interface $\mathrm{SrF}_{2}$-InP (100) a fait l'objet d'une étude XPS en limitant l'épaisseur des films à $40 \AA$. Pour ne pas perturber les liaisons chimiques aucune pulvérisation des échantillons n'a été réalisée, par contre une variation de l'angle de détection $\theta$ des photo-électrons permet de localiser les atomes émetteurs dans l'épaisseur de la structure. Les spectres a et b de la figure 2 représentent, respectivement, les réponses des électrons $3 \mathrm{~d}$ de l'indium et $2 \mathrm{~s}$ du phosphore. Les courbes $\alpha$ et $\beta$ correspondent à $\theta=90^{\circ}$ et $45^{\circ}$ par rapport à la surface de l'échantillon. Après étalonnage préalable, les pourcentages atomiques des différents éléments détectés suivant ces deux angles sont reportés dans le tableau ci-dessous. Le carbone et l'oxygène résultent sûrement d'une pollution atmosphérique responsable, nous l'avons déjà souligné, du léger défaut de fluor relevé sur ces films très minces. L'évolution des teneurs en indium et en phosphore en fonction de $\theta$ indique une accumulation de ce dernier à
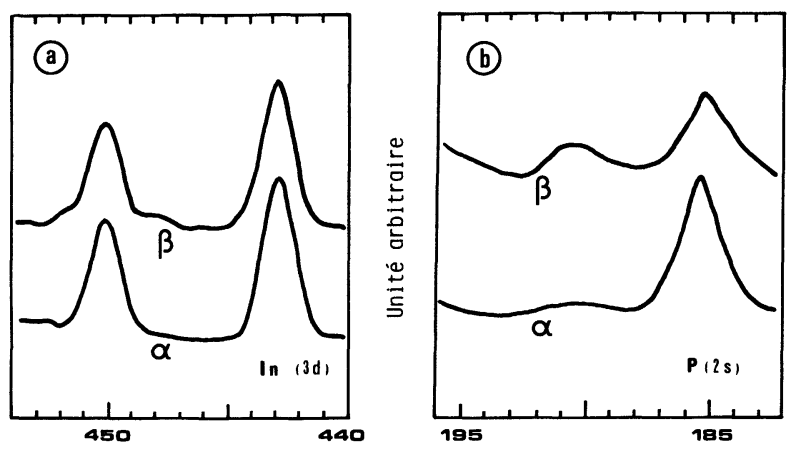

Energie de liaison (ev)

Fig. 2. - Spectres XPS : (a) des électrons 3d de l'indium et (b) des électrons $2 \mathrm{~s}$ du phosphore d'une couche de $\mathrm{SrF}_{2}$ de $40 \AA$ d'épaisseur déposée à $T_{\mathrm{S}}=573 \mathrm{~K}$ sur un substrat d'InP (100). Les courbes $\alpha$ et $\beta$ correspondent à des angles de détection $\theta=90^{\circ}$ et $45^{\circ}$.

[XPS spectra, (a) and (b) for 3d and 2s electrons of indium and phosphorus respectively for a $40 \AA$ thick $\mathrm{SrF}_{2}$ layers deposited at $573 \mathrm{~K}$ on a (100) InP crystal. Curves $\alpha$ and $\beta$ correspond to an angle of detection of $90^{\circ}$ and $45^{\circ}$.]

Tableau I. - Concentrations atomiques (en \%) des différents éléments détectés par XPS sur une structure $\mathrm{SrF}_{2}(40 \AA)$ - InP (100), pour deux angles d'émission $\theta$.

[Atomic concentration (\% At) of the different elements detected by XPS on a $\mathrm{SrF}_{2}(40 \AA)$ - InP (100) structure for two $\theta$ angles of emission.]

\begin{tabular}{l|cccccc} 
& $\mathrm{C}(1 \mathrm{~s})$ & $\mathrm{O}(1 \mathrm{~s})$ & $\mathrm{F}(1 \mathrm{~s})$ & $\mathrm{Sr}(3 \mathrm{~d})$ & $\operatorname{In}(3 \mathrm{~d})$ & $\mathrm{P}(2 \mathrm{~s})$ \\
\hline $\boldsymbol{\theta}=90^{\circ}$ & 23,6 & 7,4 & 43,1 & 21,3 & 2,7 & 1,9 \\
$\boldsymbol{\theta}=45^{\circ}$ & 28,1 & 5,2 & 41,1 & 21,9 & 1,3 & 2,4
\end{tabular}

l'interface $\mathrm{SrF}_{2}$-InP. Par ailleurs l'apparition pour $\theta=45^{\circ}$ (spectre b, $\beta$, Fig. 2) d'un pic de phosphore décalé de $5,3 \mathrm{eV}$ par rapport au pic des électrons $2 \mathrm{~s}$ du phosphore lié à l'indium met en évidence des liaisons P-F. Par contre rien de semblable n'est observé en fonction de $\theta$ sur les spectres de l'indium.

2.2 ETUDES ÉlECTRIQUES. - Les caractéristiques courant-tension $(C-V)$ des structures MIS ont été obtenues à température ambiante, en utilisant un capacimètre Boonton travaillant à $1 \mathrm{MHz}$. Le balayage en tension varie ici de $-5 \mathrm{~V}$ à $+5 \mathrm{~V}$.

Pour une structure $\mathrm{Hg}-\mathrm{SrF}_{2}$ (1 $300 \AA$ d'épaisseur) - InP $\left((100), n\left(5 \times 10^{15} \mathrm{~cm}^{-3}\right)\right)$ - AuGe, de surface utile $S=0,78 \mathrm{~mm}^{2}$, et une fréquence de balayage (F.B.) supérieure ou égale à $0,5 \mathrm{~Hz}$, les caractéristiques $C-V$ sont données par la courbe $\alpha$ de la figure 3. Elles mettent en évidence une importante modulation du potentiel de surface $\psi_{\mathrm{s}} \mathrm{du}$ semiconducteur, sans hystérésis.

Ce résultat peut être comparé au $C$ - $V$ théorique hautes fréquences (HF) de la structure (courbe $\delta$, Fig. 3), construit à partir de l'évolution de $\psi_{\mathrm{s}}$ calculée en supposant un schéma de bandes plates à l'équilibre (encart a, Fig. 3), et d'un schéma équivalent analogue à celui qui est présenté en encart b, figure $3[11,12]$. Dans ce dernier la contribution du diélectrique $(\mathrm{Ri}, \mathrm{Ci})$ peut être estimée à partir de la mesure sous tension alternative de la tangente de l'angle de perte $(\operatorname{tg} \delta)$ d'une structure M.I.M. à température ambiante et $1 \mathrm{MHz}, \operatorname{tg} \delta=0,3$.

En premier lieu on remarque que le décalage entre courbes théorique et expérimentale, $\Delta V=$ $1,7 \mathrm{eV}$, traduit la présence de charges négatives fixes dans l'isolant, ou situées à l'interface isolant-semiconducteur, dont le nombre par unité de surface peut être estimé, à partir de la relation $N=\frac{C_{i} \Delta V}{q S}$, à $5 \times 10^{11} \mathrm{~cm}^{-2}$.

A ce décalage près on note, en second lieu, que les évolutions des $C-V$ expérimentaux et théoriques présentent une similitude telle, dans les domaines d'accumulation et de déplétion, qu'il ne nous est pas possible, pour de telles fréquences, d'évaluer une densité d'états de surface dans le semi-conducteur $\left(N_{\text {SS }}\right)$ par la méthode classique de Terman. Dans ce cas il est vraisemblable que $N_{\mathrm{SS}} \approx 10^{11} \mathrm{eV}^{-1} \mathrm{~cm}^{-2}$. Ce résultat pourrait être, du moins partiellement, le fait des liaisons phosphore-fluor que nous avons mises en évidence par XPS, ayant pour effet de réduire le nombre de liaisons pendantes à la surface d'InP.

On remarque enfin que pour les fortes tensions négatives la capacité mesurée est inférieure à la capacité théorique. Un tel phénomène peut s'expliquer soit par un transfert tunnel d'électrons primitivement situés sur des états localisés de la bande interdite de l'isolant dans la bande de valence du 


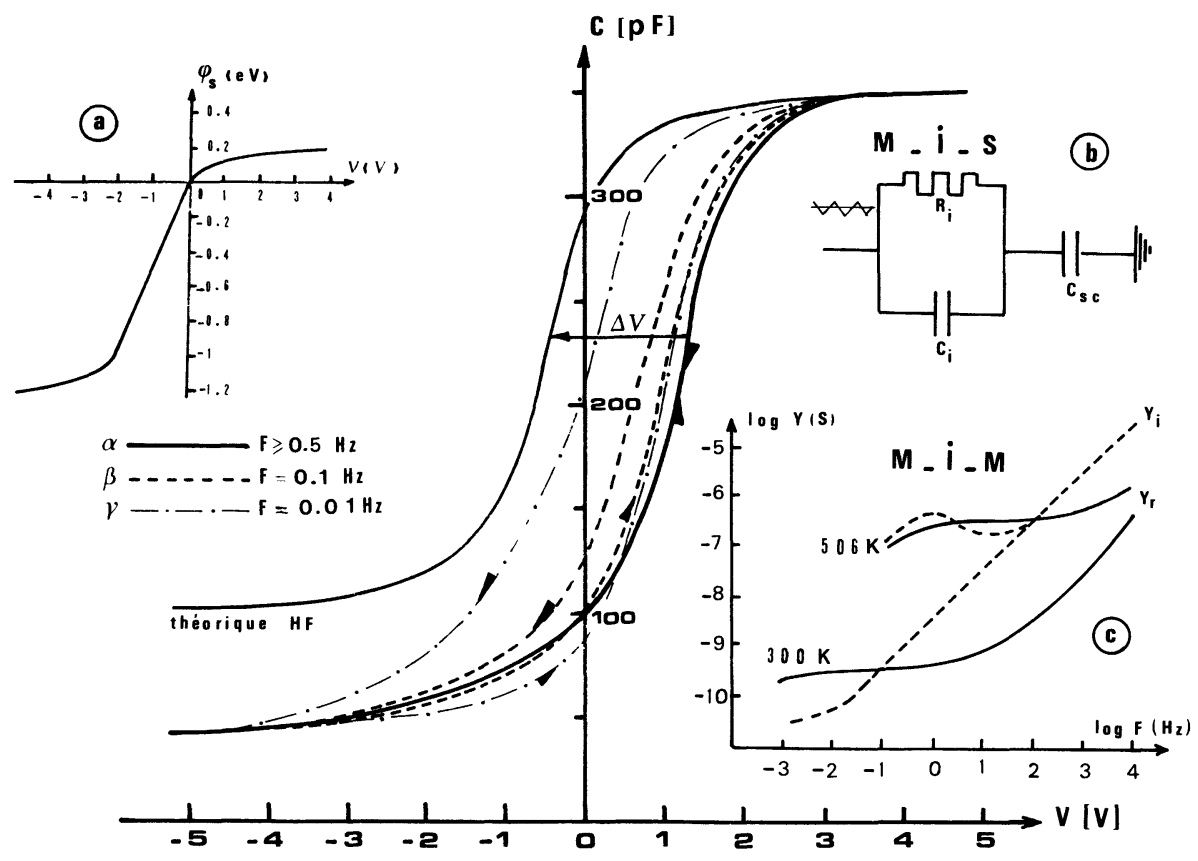

Fig. 3. - Caractéristiques $C-V$ expérimentales $\alpha, \beta$ et $\gamma$, obtenues respectivement avec une fréquence de balayage : $F . B .>0,5 \mathrm{~Hz}, F . B .=0,1 \mathrm{~Hz}$ et $F . B .=0,01 \mathrm{~Hz}$ et théorique $\delta$ d'une structure $\mathrm{Hg}_{-} \mathrm{SrF}_{2}$ (1300 $\AA$ ) - InP ((100), $\left.n .5 \times 10^{15} \mathrm{~cm}^{-3}\right)-A u G e$. Les études ont été réalisées à température ambiante; la fréquence de mesure est de $1 \mathrm{MHz}$. Les encarts $\mathrm{a}, \mathrm{b}$ et $\mathrm{c}$ représentent respectivement l'évolution du potentiel de surface du semi-conducteur, le schéma équivalent de la structure MIS et l'admittance d'une structure $\mathrm{Au}-\mathrm{SrF}_{2}-\mathrm{Au}$ à deux températures.

semi-conducteur [13-15], soit par la neutralisation d'espèces chargées mobiles.

Le deuxième phénomène semblerait ici le plus probable. En effet, comme le montre le diagramme d'admittance d'une structure $\mathrm{Au}-\mathrm{SrF}_{2}-\mathrm{Au}$, porté en encart $\mathrm{c}$, figure 3 , même si à $T=300 \mathrm{~K}$ et à basse fréquence la conduction des films de $\mathrm{SrF}_{2}$ est très faible $\left(\sigma=3 \times 10^{-13} \mathrm{~S} \mathrm{~cm}^{-1}\right)$ et leur permittivité diélectrique mesurée $\left(\varepsilon_{\mathrm{r}}=6,6\right)$ correspond bien à la valeur trouvée pour le matériau massif [16], il semble bien qu'une participation ionique soit révélée par l'évolution des parties réelle $Y_{\mathrm{R}}$ et imaginaire $Y_{\mathrm{I}}$ à $T=506 \mathrm{~K}$ [17]. Ainsi, et même si un transfert électronique peut devenir le principal responsable des fuites à travers les films de $\mathrm{SrF}_{2}$ à température ambiante, une faible participation ionique pourrait être la cause d'un défaut d'écrantage des trous dans la zone d'inversion du semi-conducteur.

Les déplacements dans l'isolant de ces mêmes porteurs lents pourraient, de plus, être la cause de l'apparition, sur les caractéristiques $C-V$ des structures MIS, d'un phénomène d'hystérésis, de plus en plus marqué au fur et à mesure que F.B. décroît. Nous donnons pour exemple les caractéristiques $C$ - $V$ obtenues à $0,1 \mathrm{~Hz}$ et $0,01 \mathrm{~Hz}$, figure $3, \beta$ et $\gamma$ respectivement. $[\alpha, \beta$ et $\gamma: 1 \mathrm{MHz}$ experimental $C-V$ at $300 \mathrm{~K}$ of a $\mathrm{Hg}$ $\mathrm{SrF}_{2}(1300 \AA)-\mathrm{n}$ type $\left(5 \times 10^{15} \mathrm{~cm}^{-3}\right) \operatorname{InP}(100)-\mathrm{AuGe}$ structure obtained with a sweep frequency $S F>0.5 \mathrm{~Hz}$, $S F=0.1$ and $0.01 \mathrm{~Hz}$ respectively and $\delta$ theoretical. The inserts $\mathrm{a}, \mathrm{b}$ and $\mathrm{c}$ represent respectively the evolution of the surface potential of the semiconductor, the equivalent diagram of the MIS structure and the admittance of a Au$\mathrm{SrF}_{2}-\mathrm{Au}$ structure for two temperatures.]

Signalons toutefois que la participation ionique ne saurait être le fait de la conduction intrinsèque du matériau qui se manifeste à des températures beaucoup plus élevées $[18,19]$. Bien que nous ayons montré que les films de $\mathrm{SrF}_{2}$ sont quasi stœchiométriques, ce transport de matière doit être le reflet de défauts dans les couches, notamment aux joints de grains. Une telle imperfection peut être considérablement réduite en abaissant, sous ultra-vide, la vitesse de condensation lors du recueil des vapeurs qui doit se traduire par une meilleure compacité des films. Des travaux en ce sens sont entrepris au Laboratoire, les résultats obtenus seront présentés ultérieurement.

\section{Etudes de GaAs et InP fluorurés.}

3.1 PRÉPARATION, CARACTÉRISATIONS PHYSICOCHIMIQUES. - La fluoruration des composés III-V (GaAs et InP) a fait l'objet d'un brevet ANVARCNRS [20]. Les substrats dégraissés sont introduits sous argon dans un réacteur en nickel qui est ensuite connecté à une rampe à fluor. La nature, l'état cristallographique et l'épaisseur des films de fluorures formés dépendent de la pression de l'agent fluorant $(P)$, de la température à laquelle sont portés les échantillons pendant la réaction $\left(T_{\mathrm{f}}\right)$ et du 
temps d'exposition $(t)$. Une étude systématique de l'influence de ces divers paramètres est actuellement en cours. Les résultats que nous présentons ici ont été obtenus avec $P=5 \mathrm{bar}, T_{\mathrm{f}}=200^{\circ} \mathrm{C}$ et des temps de fluoruration variables.

Pour des temps de fluoruration de $9 \mathrm{~h}$ dans le cas de GaAs et de $15 \mathrm{~h}$ dans le cas d'InP, les spectres R.B.S. des semi-conducteurs fluorés sont présentés figure $4 \alpha$, a et $b$ respectivement. Pour comparaison nous avons également reporté les spectres de GaAs et d'InP bruts figure $4 \beta$, a et $b$.
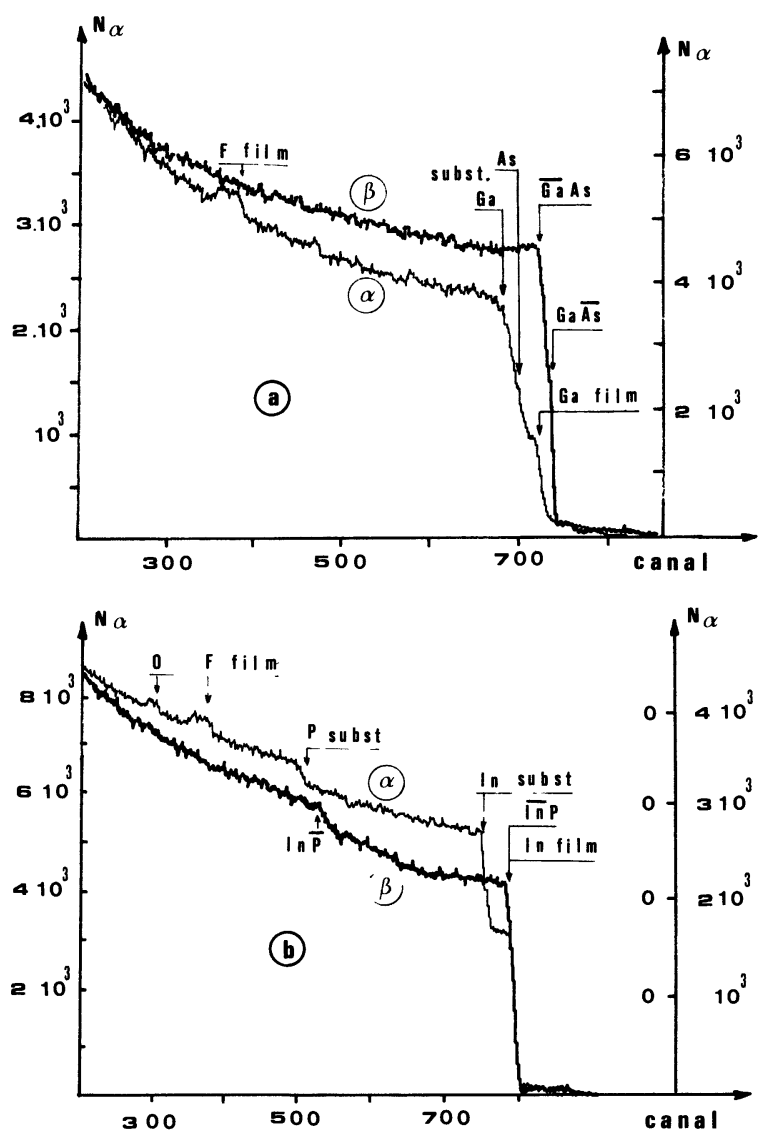

Fig. 4. - ( $\alpha)$ spectres R.B.S. de GaAs (a) et d'InP (b) fluorés ( 5 bar, $200^{\circ} \mathrm{C}, t=9$ et $15 \mathrm{~h}$ respectivement). Les spectres $\beta$ correspondent aux semi-conducteurs bruts.

[( $\alpha)$ R.B.S. spectra of fluorinated GaAs (a) and InP (b) ( 5 bar, $200{ }^{\circ} \mathrm{C}, t=9$ and $15 \mathrm{~h}$ respectively). The spectra $\beta$ corresponds to the single crystal.]

Dans les deux cas, on remarque qu'à la surface et dans le volume des couches de fluorures obtenues, d'épaisseur voisine de $1000 \AA$, seuls les cations III$B$ ( $G$ et In respectivement pour GaAs et InP fluorurés) ont été détectés par R.B.S. (début des spectres au canal du gallium dans le premier cas, recul du front de phosphore dans le second). Les éléments V-B fluorés, volatils dans les conditions de préparation adoptées, ont vraisemblablement été éliminés avec l'agent fluorant en fin de réaction. On note de plus que les fronts de montée des signaux relatifs aux substrats sont relativement abrupts montrant ainsi que les zones d'interdiffusion ont une épaisseur très faible. La teneur en fluor rapportée à un atome III-B a été déterminée par comparaison de l'intensité des divers signaux. Avec une très forte imprécision, due à la superposition des spectres des couches et des substrats, on obtient $N_{\mathrm{F}} / N_{\mathrm{Ga}}=$ $2 ; 8 \pm 0,25$ et $N_{\mathrm{F}} / N_{\text {In }}=2,8 \pm 0,3$. Il semble donc que, dans les conditions de fluoruration de GaAs et d'InP adoptées, les formules chimiques du volume des films obtenus soient $\mathrm{GaF}_{3}$ et $\mathrm{InF}_{3}$ respectivement. Dans le cas d'InP fluoré, l'observation sur le spectre R.B.S., au canal 310, d'un pic étroit, de faible intensité, peut traduire une adsorption de vapeur d'eau par le fluorure lors de la mise à l'air des échantillons. On notera toutefois qu'après un premier contact avec l'atmosphère, les résultats présentés restent stables dans le temps.

Pour tenter de décrire les interfaces couchessubstrats de telles structures, une étude XPS a été réalisée sur des échantillons obtenus avec des temps de fluoruration très courts $(20 \mathrm{~min})$. Dans ce cas l'épaisseur des films de fluorures formés est inférieure à $100 \AA$. Nous reportons figure $5 \mathrm{a}, \mathrm{b}, \mathrm{c}$ et $\mathrm{d}$ les spectres d'émission des électrons $3 \mathrm{~d}$ de As et de
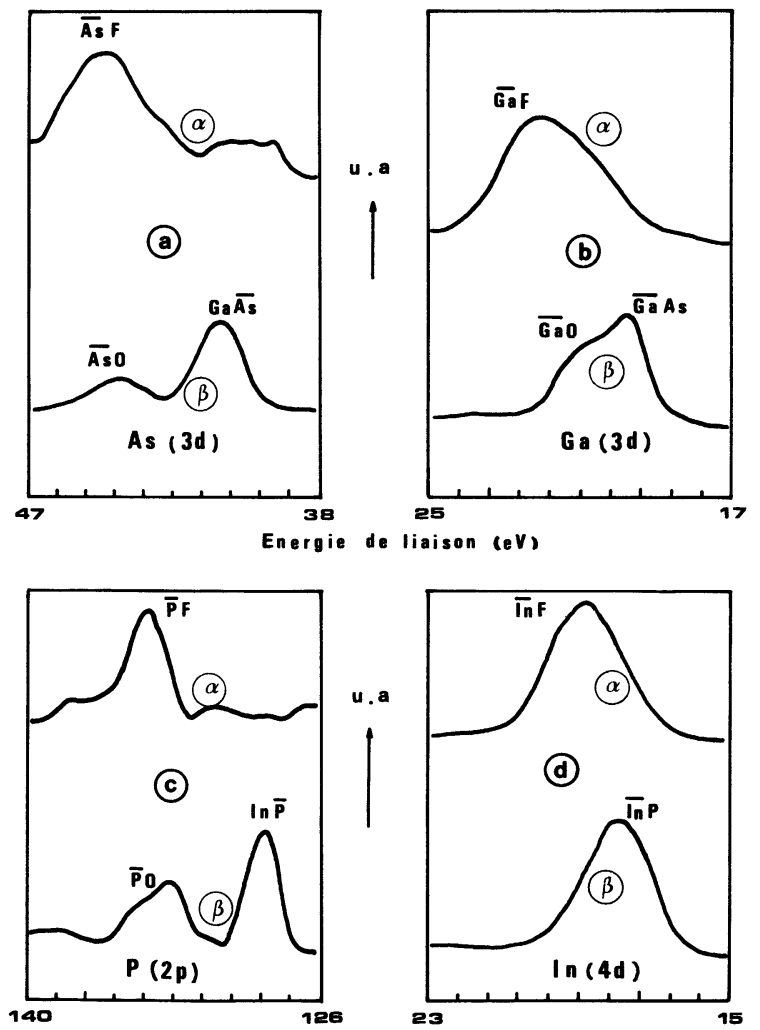

Fig. 5. - Spectres d'émission des électrons $3 \mathrm{~d}$ de l'arsenic (a), 3d du gallium (b), $2 \mathrm{p}$ du phosphore (c) et $4 \mathrm{~d}$ de l'indium (d) de monocristaux de GaAs et d'InP fluorés ( $\alpha$ ) et bruts $(\beta)$.

[Emission spectra of the electrons 3d of arsenic (a), 3d of gallium (b), 2p of phosphorus (c) and $4 d$ of indium (d) of fluorinated GaAs and $\operatorname{InP}(\alpha)$ and of single crystals $(\beta)$.] 
$\mathrm{Ga}, 2 \mathrm{p}$ de $\mathrm{P}$ et $4 \mathrm{~d}$ d'In respectivement pour des échantillons de GaAs et InP fluorés $(\alpha)$ et n'ayant subi aucun traitement $(\beta)$. Si on observe bien les liaisons $\mathrm{Ga}-\mathrm{F}$ et In-F, comme le laissaient présager les études R.B.S., nous devons de plus signaler ici la détection des éléments $\mathrm{V}$-B (As et $\mathrm{P}$ ), qui montre leur subsistance près de l'interface, et leurs liaisons avec le fluor. L'élargissement des pics, côté basses énergies, indique des liaisons avec de l'oxygène dont la présence est inévitable dans le cas de films aussi minces exposés à l'air.

3.2 ETUdes ÉlectriQues. - Toujours pour une fréquence de meure de $1 \mathrm{MHz}$, les caractéristiques expérimentales (courbes a), obtenues à température ambiante et jusqu'à une fréquence de balayage de $0,01 \mathrm{~Hz}$, et théoriques HF (courbe b) de structures MIS, Hg-GaAs ((100), $\left.n \times 10^{16} \mathrm{~cm}^{-3}\right)$ fluoré $(5 \mathrm{bar}$, $\left.200{ }^{\circ} \mathrm{C}, 15 \mathrm{~h}\right)-\mathrm{AuGe}$ et $\mathrm{Hg}-\mathrm{InP}((100), n .5 \times$ $10^{15} \mathrm{~cm}^{-3}$ ) fluoré $\left(5 \mathrm{bar}, 200{ }^{\circ} \mathrm{C}, 36 \mathrm{~h}\right)-\mathrm{AuGe}$, sont reportées figures 6 et 7 respectivement. Dans ces conditions de fluoruration les épaisseurs des films de fluorures formés sont de $1500 \AA$ et $2300 \AA$ respectivement. La surface de l'électrode de mesure est ici égale à $0,432 \mathrm{~mm}^{2}$.

Dans les deux cas, ces résultats mettent en évidence une importante fluctuation de la capacité des structures, soit donc une importante modulation du potentiel de surface du semi-conducteur, en fonction de la tension appliquée, pratiquement sans hystérésis jüsqu'à une fréquence de balayage de $0,01 \mathrm{~Hz}$.

Comme dans le cas précédent le décalage entre les courbes expérimentales et théoriques $\Delta V=1,2 \mathrm{~V}$ et $4,8 \mathrm{~V}$ respectivement pour $\mathrm{GaAs}$ et InP fluorés, indique la présence de charges fixes dans l'isolant; leurs nombres par unité de surface de jonction sont estimés à $4,6 \times 10^{11} \mathrm{~cm}^{2}$ pour $\mathrm{GaAs}$ fluoré et à $1,25 \times 10^{12} \mathrm{~cm}^{-2}$ pour InP fluoré.

Par comparaison des $C$ - $V$ théoriques et expérimentaux on a pu ici, à partir de la méthode de Terman, estimer les densités d'états de suŕface dans les domaines d'accumulation et de déplétion des semiconducteurs à $5 \times 10^{11} \mathrm{eV}^{-1} \mathrm{~cm}^{-2}$ et $10^{12} \mathrm{eV}^{-1}$ $\mathrm{cm}^{-2}$ pour $\mathrm{GaAs}$ fluoré et InP fluoré respectivement. Pour GaAs fluoré on remarque toutefois, sur la caractéristique expérimentale, un point anguleux qui semble indiquer un blocage partiel du niveau de Fermi du semi-conducteur. Cette observation pourrait être la conséquence d'une forte densité d'états situés dans sa bande interdite à environ $1 \mathrm{eV}$ audessous de sa bande de conduction. Dans le cas d'InP le passage en déplétion profonde pour de fortes tensions négatives révèle à nouveau un défaut d'écrantage des trous dans le semi-conducteur.

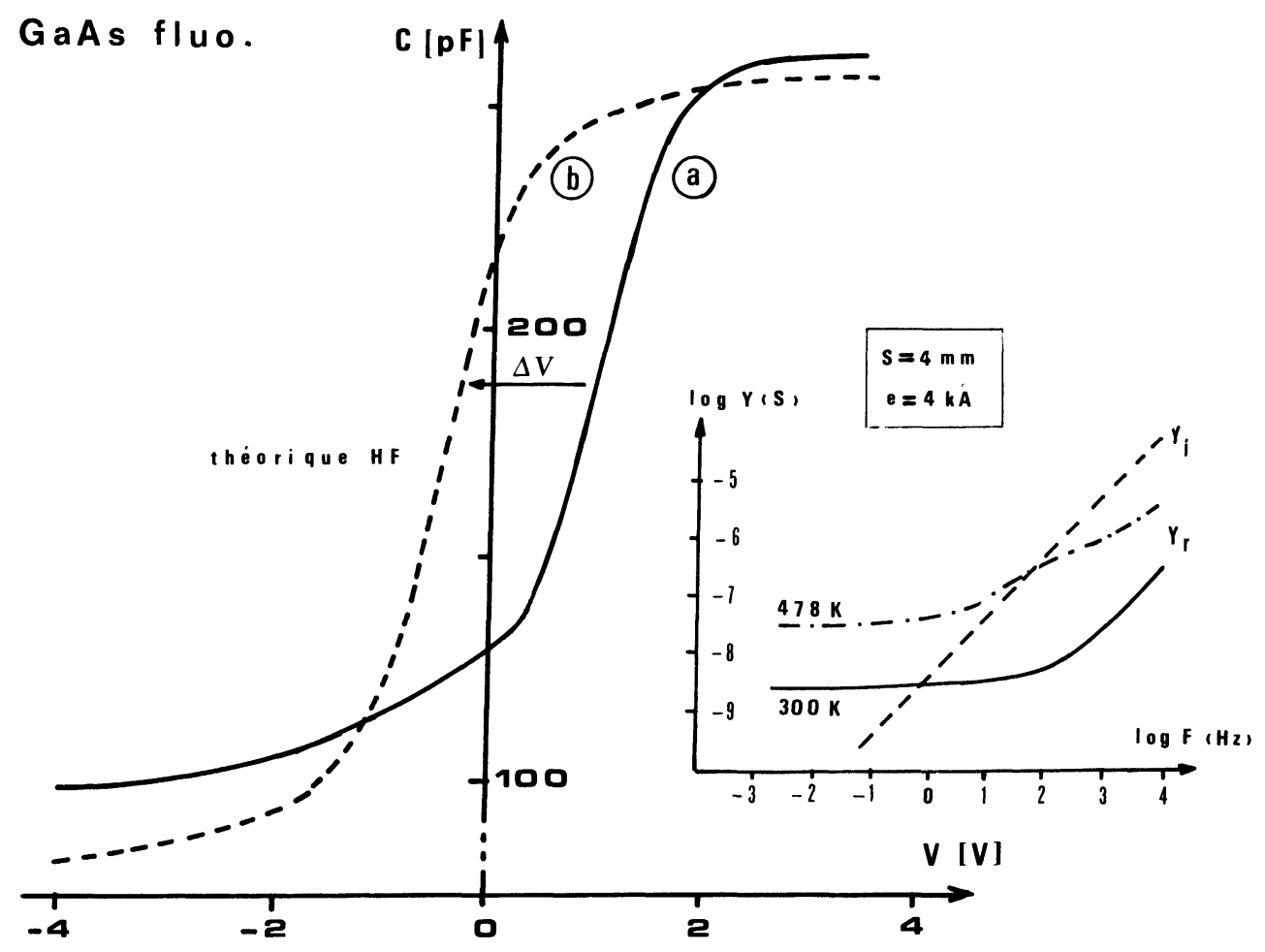

Fig. 6. - Caractéristiques $C-V$ à $1 \mathrm{MHz}$ expérimentale $(F . B .=0,01 \mathrm{~Hz})$ (a) et théorique (b) d'une structure $\mathrm{Hg}$ GaAs $\left((100), n \cdot 10^{16} \mathrm{~cm}^{-3}\right)$ fluoré $\left(5 \mathrm{bar}, 200^{\circ} \mathrm{C}, 15 \mathrm{~h}\right)$ AuGe. En encart est reportée l'admittance, pour deux températures de mesure, d'une structure $\mathrm{Au}-\mathrm{GaF}_{3}-\mathrm{Au}$ obtenue par sublimation thermique sous vide.
[1 $\mathrm{MHz}$ experimental $(S . F=0.01 \mathrm{~Hz})$ (a) and theoretical (b) $\mathrm{C}-\mathrm{V}$ characteristics of a $\mathrm{Hg}$-fluorinated $\left(5 \mathrm{bar}, 200^{\circ} \mathrm{C}\right.$, $15 \mathrm{~h}) \mathrm{GaAs}\left((100), n \cdot 10^{16} \mathrm{~cm}^{-3}\right)$ structure. The insert shows the admittance of a $\mathrm{Au}-\mathrm{GaF}_{3}-\mathrm{Au}$ structure, obtained by sublimation under vacuum, for two temperatures.] 


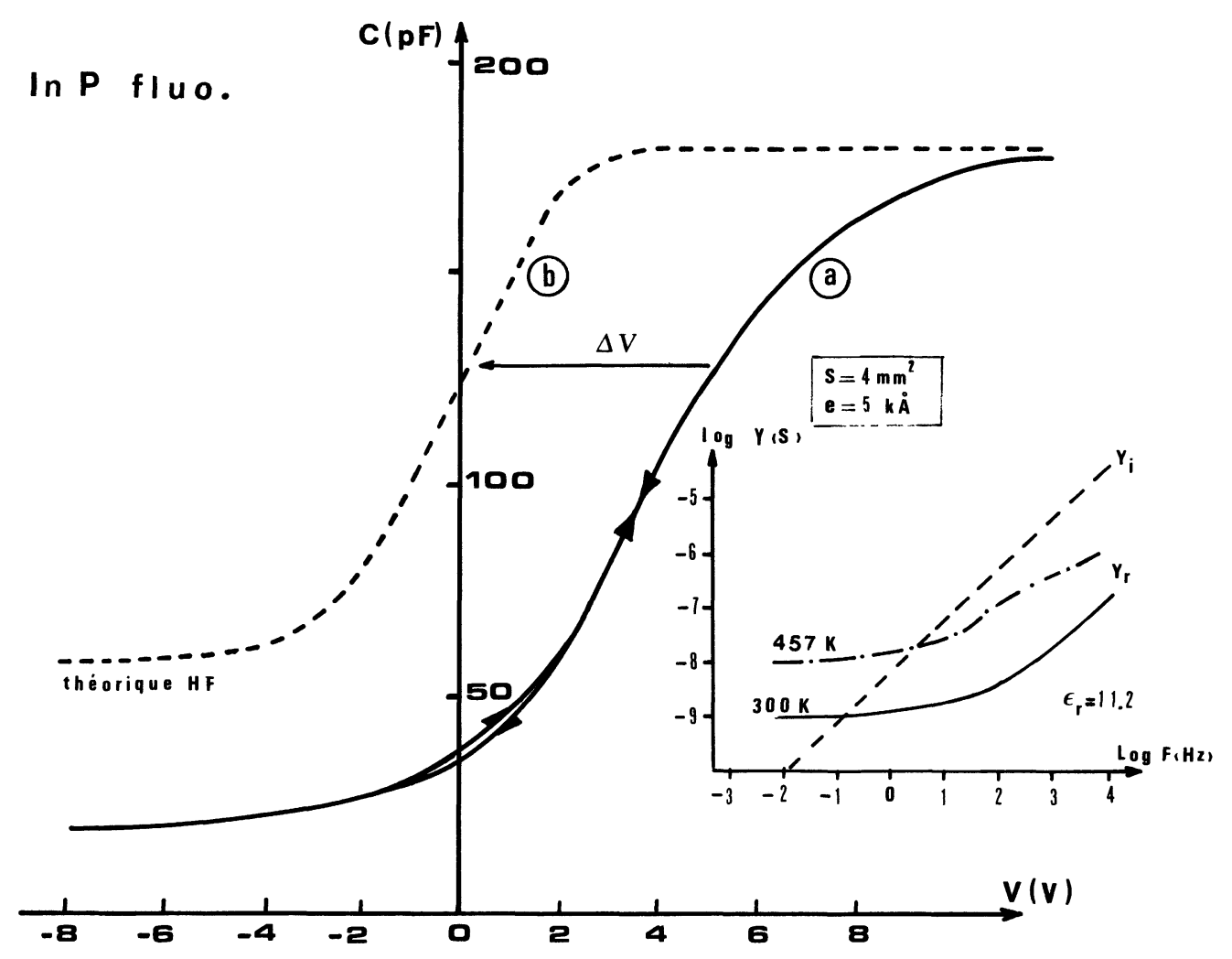

Fig. 7. - Caractéristiques $C-V$ à $1 \mathrm{MHz}$ expérimentale $(F . B .=0,01 \mathrm{~Hz})(\mathrm{a})$ et théorique (b) d'une structure $\mathrm{Hg}$ InP $\left((100), n .5 \times 10^{15} \mathrm{~cm}^{-3}\right)$ fluoré $\left(5 \mathrm{bar}, 200^{\circ} \mathrm{C}, 36 \mathrm{~h}\right)$ AuGe. En encart est reportée l'admittance, à température ambiante, d'une structure $\mathrm{Au}-\mathrm{InF}_{3}-\mathrm{Au}$ obtenue par sublimation thermique sous vide.

Notons enfin que, dans les conditions actuelles de fabrication, des instabilités sur les caractéristiques $C-V$ des structures MIS GaAs fluoré nous ont fait limiter le balayage en tension de plus ou moins $4 \mathrm{~V}$.

Malgré ces imperfections ces résultats sont encourageants notamment dans le cas de GaAs. Pour expliquer que, par fluoruration, une aussi faible densité d'états puisse être observée sur une large plage d'énergie de la bande interdite, à la surface de ce semi-conducteur, nous avons émis deux hypothèses : i) rejetant l'interface isolant-semi-conducteur dans le volume de ce dernier, il doit être pratiquement exempt d'oxydes natifs dont la présence semble être une cause essentielle du blocage du niveau de Fermi, ii) les liaisons pendantes, insatisfaites dans le cas d'un dépôt, peuvent être ici partiellement neutralisées par le fluor, comme semblent le montrer les résultats XPS.

Pour InP, par contre, les piètres résultats obtenus ici, en comparaison de ceux que nous avons présentés pour le couple $\mathrm{SrF}_{2}$-InP, pourraient être le fait de la présence d'oxygène, que les analyses R.B.S. ont révélée. Une modification des conditions de fluoruration peut, nous l'espérons, minimiser cette imperfection.
[1 $\mathrm{MHz}$ experimental $(S . F=0.01 \mathrm{~Hz})(\mathrm{a})$ and theoretical (b) $\mathrm{C}-\mathrm{V}$ characteristics of a $\mathrm{Hg}$-fluorinated $\left(5 \mathrm{bar}, 200^{\circ} \mathrm{C}\right.$, $36 \mathrm{~h}) \operatorname{InP}\left((100), n .5 \times 10^{15} \mathrm{~cm}^{-3}\right)$ structure. The insert shows the admittance, at room temperature, of a $\mathrm{Au}$ $\mathrm{InF}_{3}-\mathrm{Au}$ structure prepared by sublimation under vacuum.]

Bien que des films résultant d'une fluoruration puissent présenter des différences sensibles avec des couches minces du même composé obtenues par sublimation thermique sous vide, même si des études R.B.S. et XPS semblent montrer que ces dernières présentent la stœchiométrie attendue, des structures $\mathrm{Au}-\mathrm{GaF}_{3}(4000 \AA)$ - Au et $\mathrm{Au}-\mathrm{InF}_{3}(5000 \AA)$ - Au, de surface utile $4 \mathrm{~mm}^{2}$ ont été réalisées par cette technique de préparation pour caractériser les isolants. Des spectres d'admittance obtenus, reportés en encart figures 6 et 7 , on peut : d'une part déduire la permittivité diélectrique des fluorures, pour $\mathrm{GaF}_{3}, \varepsilon_{\mathrm{r}}=10,8$, pour InP, $\varepsilon_{\mathrm{r}}=11,2$; d'autre part remarquer que, si dans le deux cas des fuites mettent en évidence une imperfection des couches, l'évolution de $Y_{\mathrm{R}}$ en fonction de la température ne saurait ici être interprétée en terme de transport ionique prédominant. Si nous pouvons transposer cette observation au cas des structures MIS obtenues par fluoruration, elle pourrait expliquer la quasi-absence d'hystérésis que nous venons de noter sur les $C-V$, même jusqu'à une fréquence de balayage de $0,01 \mathrm{~Hz}$.

Dans ce cas le passage en déplétion profonde des MIS InP fluorées pourrait ici être, à nouveau, le fait 
de la présence d'oxydes dans les films de fluorure qui se traduirait par la présence d'états localisés situés dans la bande interdite de l'isolant.

\section{Conclusion.}

Nous avons montré que les structures $\mathrm{SrF}_{2}$ (préparées par sublimation thermique sous vide classique) InP se caractérisent par une très faible densité d'états d'interface. Dans ce cas, cet isolant, ainsi obtenu, semblerait devoir être un bon candidat pour réaliser une bonne passivation d'InP. Par contre, dans ces conditions de préparation, sa conductivité ionique extrinsèque semble interdire d'envisager son utilisation pour la réalisation de transistors à effet de champ. Par abaissement de leur vitesse de condensation sous ultra-vide, une meilleure cristallisation des couches devrait réduire ces imperfections. Des travaux en ce sens sont en cours au Laboratoire.

Par ailleurs il est établi qu'une fluoruration de GaAs et d'InP, conduisant à la formation de films isolants, encapsulants et ne présentant pas de transport ionique, pourrait ouvrir une nouvelle voie intéressante pour l'obtention de composants III-V à effet de champ. En effet cette technique semble pouvoir permettre la modulation du potentiel de surface de GaAs et d'InP, sans hystérésis jusqu'à des très faibles fréquences de balayage. Une amélioration de la qualité de ces couches doit être obtenue par une étude, entreprise au Laboratoire, de la cinétique de fluoruration des semi-conducteurs en fonction de la nature et de la pression de l'agent fluorant de la température des substrats lors de la réaction et des temps d'exposition.

\section{Remerciements.}

Nous tenons à remercier Monsieur D. Lecrosnier du Centre d'Etudes des Télécommunications (CNET Lannion B) pour ses précieux conseils et son soutien matériel et Monsieur J. Salardenne pour les discussions scientifiques qu'il a bien voulu nous accorder.

Bibliographie

[1] Hasegawa, H., Forward, K. E. and Hastnagel, H. L., Appl. Phys. Lett. 26 (1975) 567.

[2] Hayashi, H., Kiruchi, K. and Yamaguchi, Appl. Phys. Lett. 37 (1980) 404.

[3] VAN StaA, P., ROMBACH, H. and Kassing, R., $J$. Appl. Phys. 54 (1983) 4014.

[4] Journées d'Etude sur l'Epitaxie et la Passivation des Composés III-V, Plestin-les-Grèves 23-24 avril 1986, Revue Le Vide, Les Couches Minces (mars-avril 1986).

[5] Siskos, S., Fontaine, C. and Munoz-Yague, A., J. Appl. Phys. 50 (1984) 1642.

[6] Ishiwara, H., Tsutsui, K., Azano, T. and FuruKaWA, S., Jpn J. Appl. Phys., Part 2 (1984) 23.

[7] Sullivan, P. W., Farrow, R. F. C. and Jones, G. R., J. Cryst. Growth 60 (1982) 403.

[8] Tu, C. W., Sheng, T. Y., Marcrander, A. T., Phillips, J. M. and Guggenheim, H. J., J. Vac. Sci. Technol. B 2 (1984) 24.

[9] Barrière, A. S., Couturier, G., Gevers, G., GranNEC, J., RicARD, H. and SRIBI, C., Surf. Sci. 168 (1986) 688.

[10] Barrière, A. S., Chaouki, A., Gevers, G., Guégan, H., Sribi, C., Bertault, D., Haw, C. and ALNOT, P., «A characterization of $\mathrm{SrF}_{2}$ thin films », Thin Solid Films, proposée pour publication, avril 1987.

[11] Grove, A. S., Show, E. H.; Deal, B. E. and SAH, C. T., J. Appl. Phys. 35 (1964) 2458.

[12] Grove, A. S., Deal, B. E., SNow, E. H. and SaH, C. T., Solid State Electron. 8 (1965) 145.

[13] Card, H. C. and Rhoderick, E. H., J. Phys. D 4 (1971) 1602.

[14] Goodnick, S. M., Hwang, T. and WILMSEN, C. W., Appl. Phys. Lett. 44 (1984) 453.

[15] Gerb, K. M., GOOdNICK, S. M., LIN, D. Y., GANN, R. G., WilmSEN, C. W. and WAGER, J. F., J. Vac. Sci. Technol. B 2 (1984) 516.

[16] Tomiki, T. and MiYata, T., J. Phys. Soc. Jpn 27 (1969) 656.

[17] Couturier, G., Thèse de Doctorat ès Sciences $\mathrm{n}^{\circ}$ 726, Université de Bordeaux I (1982).

[18] Archer, J. A., Chadwick, A. V., JACK, I. R. and ZEQUIRI, B., Solid State Ionics 9-10 (1983) 505.

[19] NAGEL, L. E. and O'KeEFE, in Fast ion transport in solid, Ed W. Van Gool (North Holland) 1972, p. 165.

[20] Tressaud, A., Grannec, J., Barrière, A. S., Lozano, L., Couturier, G. et SÉGuelong, T., Brevet ANVAR-CNRS n ${ }^{\circ} 8605957$ du 24-041986. 